\title{
Nonoperative Treatment With Antibiotics Versus Surgery for Acute Nonperforated Appendicitis in Children
}

\author{
A Pilot Randomized Controlled Trial
}

\author{
Jan F. Svensson, MD, ${ }^{*} \dagger$ Barbora Patkova, MD ${ }^{*} \dagger$ Markus Almström, MD,${ }^{*} \dagger$ Hussein Naji, MD, ${ }^{*} \dagger$ \\ Nigel J. Hall, MD, PhD, $\ddagger \S$ Simon Eaton, PhD, $\ddagger \|$ Agostino Pierro, MD, PhD, § and Tomas Wester, MD, PhD* $\dagger$
}

\begin{abstract}
Objective: The aim of this study was to evaluate the feasibility and safety of nonoperative treatment of acute nonperforated appendicitis with antibiotics in children.

Methods: A pilot randomized controlled trial was performed comparing nonoperative treatment with antibiotics versus surgery for acute appendicitis in children. Patients with imaging-confirmed acute nonperforated appendicitis who would normally have had emergency appendectomy were randomized either to treatment with antibiotics or to surgery. Follow-up was for 1 year. Results: Fifty patients were enrolled; 26 were randomized to surgery and 24 to nonoperative treatment with antibiotics. All children in the surgery group had histopathologically confirmed acute appendicitis, and there were no significant complications in this group. Two of 24 patients in the nonoperative treatment group had appendectomy within the time of primary antibiotic treatment and 1 patient after 9 months for recurrent acute appendicitis. Another 6 patients have had an appendectomy due to recurrent abdominal pain $(n=5)$ or parental wish $(\mathrm{n}=1)$ during the follow-up period; none of these 6 patients had evidence of appendicitis on histopathological examination.

Conclusions: Twenty-two of 24 patients (92\%) treated with antibiotics had initial resolution of symptoms. Of these 22 , only 1 patient (5\%) had recurrence of acute appendicitis during follow-up. Overall, $62 \%$ of patients have not had an appendectomy during the follow-up period. This pilot trial suggests that nonoperative treatment of acute appendicitis in children is feasible and safe and that further investigation of nonoperative treatment is warranted.
\end{abstract}

Keywords: antibiotics, appendicitis, children, randomized controlled trial, surgery

(Ann Surg 2014;00:1-5)

A cute appendicitis is the most common disease requiring emergency surgical treatment in children. Traditionally, the standard

From the *Department of Pediatric Surgery, Astrid Lindgren Children's Hospital, Karolinska University Hospital, Stockholm, Sweden; †Department of Women’s and Children's Health, Karolinska Institutet, Stockholm, Sweden; $\ddagger$ Surgery Unit, UCL Institute of Child Health, London, United Kingdom; §Division of General and Thoracic Surgery, The Hospital for Sick Children, Toronto, Ontario, Canada; and \|Department of Pediatric Surgery, Great Ormond Street Hospital, London, United Kingdom.

The trial is registered at ClinicalTrials.gov: No. NCT01572558

Disclosure: Supported by the Crown Princess Lovisa's Foundation and the Hirsch Foundation grants. The funders had no role in study design, data collection/analysis, and decision to publish the manuscript.

The authors declare no conflicts of interest.

This is an open-access article distributed under the terms of the Creative Commons Attribution-NonCommercial-NoDerivitives 3.0 License, where it is permissible to download and share the work provided it is properly cited. The work cannot be changed in any way or used commercially.

Reprints: Jan F. Svensson, MD, Department of Pediatric Surgery, Astrid Lindgren Children's Hospital, Karolinska University Hospital, Q3:03, 17176 Stockholm, Sweden. E-mail: jan.f.svensson@karolinska.s.

Copyright (C) 2014 by Lippincott Williams \& Wilkins

ISSN: 0003-4932/14/00000-0001

DOI: $10.1097 /$ SLA.0000000000000835 treatment of acute appendicitis has been appendectomy. However, there is growing interest in nonoperative treatment of acute nonperforated appendicitis with antibiotics. Several randomized controlled trials (RCTs) have been performed in adults and these have also been subjected to meta-analysis. Data suggest that antibiotic treatment may be an effective treatment modality for adults with acute nonperforated appendicitis and that approximately $75 \%$ of patients may not need appendectomy at all, either during initial illness or during the first year of follow-up. ${ }^{1}$ However, a recent Cochrane review concluded that further well-designed RCTs were needed. ${ }^{2}$

In children, although there have been several studies of initial conservative treatment of perforated appendicitis, ${ }^{1,3,4}$ data on conservative treatment of nonperforated acute appendicitis in children are scanty. The only comparative published study was retrospective and had unclear diagnostic and treatment criteria. ${ }^{5}$ Of note, there have been no RCTs investigating nonoperative treatment of acute nonperforated appendicitis in children.

As a prelude to a large RCT investigating the efficacy of nonoperative treatment of acute nonperforated appendicitis in children, we designed a pilot RCT to inform our future planned study. The objectives of this pilot study were (i) to evaluate the feasibility of recruiting children with acute appendicitis to an RCT comparing nonoperative treatment with appendectomy; (ii) to evaluate the safety of nonoperative treatment with antibiotics of acute nonperforated appendicitis in children; and (iii) to generate pilot data to inform our future planned efficacy study.

\section{METHODS}

\section{Trial Design}

This was a pilot trial comparing nonoperative treatment (antibiotics) and surgery for acute nonperforated appendicitis in children. The diagnosis was made with the combination of clinical findings and imaging. All children underwent abdominal ultrasound scan, and a computed tomographic (CT) scan was performed when there was diagnostic uncertainty. Age, sex, duration of symptoms, body temperature, and C-reactive protein, white blood cell, and neutrophil concentrations at admission were recorded.

\section{Participants}

All children between 5 and 15 years of age with a clinical diagnosis of acute appendicitis that before the trial would have been subjected to an appendectomy, including those with an appendicolith, were eligible. Exclusion criteria were (i) suspicion of perforated appendicitis on the basis of generalized peritonitis; (ii) an appendiceal mass, diagnosed by clinical examination and/or imaging; or (iii) previous nonoperative treatment of acute appendicitis.

\section{Study Setting}

The study was conducted at the Astrid Lindgren Children's Hospital, Karolinska University Hospital, Stockholm, Sweden. This 
is the only hospital with a pediatric surgical service within the greater Stockholm area and serves a population of approximately 2.5 million inhabitants.

\section{Interventions}

Enrollment in this study was after the attending pediatric surgeon had made a diagnosis of acute appendicitis, the patients and their family had received oral and written information regarding the trial, and the patients and their family had provided written informed consent to participate. Children with acute nonperforated appendicitis were randomly allocated to either appendectomy or nonoperative treatment with antibiotics. All patients allocated to surgery received preoperative antibiotic prophylaxis with $20 \mathrm{mg} / \mathrm{kg}$ of metronidazole. Further antibiotic treatment in this group depended on the severity of appendicitis in accordance with institutional practice. Cases of simple or phlegmonous appendicitis received no further antibiotics, those with gangrenous appendicitis received 24 hours of intravenous trimethoprim/sulfametoxazol/metronidazole, and those with perforated appendicitis received at least 3 days of intravenous trimetho$\mathrm{prim} / \mathrm{sulfametoxazol} / \mathrm{metronidazole}$, depending on clinical course. The modality of surgery (open or laparoscopic) was not stipulated in the trial protocol.

Children allocated to antibiotic treatment were given intravenous meropenem $(10 \mathrm{mg} / \mathrm{kg} \times 3$ per 24 hours $)$ and metronidazole $(20 \mathrm{mg} / \mathrm{kg} \times 1$ per 24 hours $)$ for at least 48 hours. Once the child was clinically well and tolerating oral intake, the treatment was changed to oral ciprofloxacin $(20 \mathrm{mg} / \mathrm{kg} \times 2$ per 24 hours $)$ and metronidazole (20 $\mathrm{mg} / \mathrm{kg} \times 1$ per 24 hours) for another 8 days. The protocol stipulated that children should be kept nil by mouth for the first 24 hours, but in practice, we found this hard to enforce as children were clinically well and often demanded to drink and eat earlier. Criteria for discharge were established a priori and applied to both treatment groups equally. They were as follows: afebrile for 24 hours, with or without oral antibiotics, adequate pain relief on oral analgesia, tolerating a light diet, and mobile.

\section{OUTCOMES}

The primary outcome was the proportion of children in each group achieving "resolution of symptoms without significant complications." This outcome was chosen as it was applicable to both treatment arms and also because this constitutes a pragmatic goal for a patient coming to the hospital with appendicitis. Significant complications were defined as length of stay more than 7 days, abscess formation, the need for surgery within 48 hours in the antibiotic group, recurrence of appendicitis within 3 months, and negative appendectomy. Secondary outcomes measured were time from randomization to discharge, complications (wound infection, wound dehiscence, diarrhea, etc), and recurrent appendicitis within 1 year of randomization. To monitor children recruited into the study and to allow collection of a full data set, all participants were seen in the outpatient clinic at 4 to 6 weeks after discharge, with further follow-up visits at 3 and 12 months after randomization. Because we encountered difficulties getting the patients to return to the outpatient clinic at 1 year after randomization, we accepted a telephone interview with one of the parents as 1-year follow-up. The specific purpose of this 1-year follow-up was to identify episodes of recurrent appendicitis and any children who had undergone appendectomy due to recurrent symptoms or parental request at another center. We do not believe that conducting this review by telephone as opposed to in person results in significant bias for this particular outcome. As a result, we had 1 -year outcomes on all patients enrolled in the trial.

Total cost of treatment was calculated in a pragmatic way, as reimbursement methods differ between different countries and systems. Total cost per participant was calculated as a fee per day of in-hospital care, a fee for use of the operating room, and the cost of a course of intravenous and oral antibiotics for the nonoperative treatment group. Total costs that include cost for the initial hospital stay for both treatment groups and cost for any additional admission as applicable are presented.

\section{Sample Size}

As this was a pilot trial, we did not perform a power calculation. On the basis of our yearly caseload of approximately 400 cases and estimated recruitment of one third of eligible cases, we aimed to enroll 50 patients within a 6-month period.

\section{Randomization}

Allocation to groups (1:1 ratio) was made via weighted minimization at the time of enrollment in the study using the following criteria: age (5-10 years or 11-15 years), sex (male or female), and duration of symptoms ( $<48$ or $>48$ hours). All factors were weighted equally. Randomization was performed using a computer-based randomization program (Simin v 6.0; Institute of Child Health, London), which allowed complete concealment of randomization sequence.

\section{Blinding}

As this was a pilot trial comparing surgery and nonoperative treatment with antibiotics, it was not considered possible or ethical to blind patients, parents, or surgeons.

\section{Statistical Methods}

Data are presented as the proportion of participants or median (range). Data were compared using the Mann-Whitney $U$ test or the Fisher exact test as appropriate, using IBM SPSS Statistics version 22. This trial is reported in accordance with the CONSORT statement. ${ }^{6,7}$

\section{Ethical Approval}

The study was approved by the Regional Ethics Review Board (reference No. 2011/1234-31/4).

\section{RESULTS}

The trial opened on February 7, 2012, and the final participant was enrolled on October 17 the same year. One-year follow up for the cohort was completed on October 25, 2013. During the trial period, 225 children with a clinical diagnosis of acute appendicitis that before the trial would have been subjected to an appendectomy were seen at our institution. A total of 174 children were not enrolled in the trial for reasons shown in Figure 1. In addition to the defined exclusion criteria and parental nonagreement to participate, 2 children were excluded on the basis of CT findings, one with a suspicion of a carcinoid tumor of the appendix and one in whom it was impossible to differentiate between appendicitis and a Meckel diverticulitis. Overall, 52 of the 129 children $(40 \%)$ whose parents were asked whether they would consent to their child being in the trial agreed. After agreement to participate, there was failure of the computer randomization program affecting 1 case (this child was not included in the study) and in 1 case parents withdrew consent to participate in the study after allocation of treatment. This child was withdrawn from the study. To account for these 2 cases, additional participants were recruited to reach the target sample size of 50 .

Participants had similar demographic and admission characteristics both to those children whose parents declined participation and to those children who were not invited to participate (Table 1) except that the proportion of children with symptom duration of less than 48 hours was significantly lower in the group of participants who were not offered to participate in the trial. The reason for this is unclear, although it is possible that surgeons felt that there was a clearer 


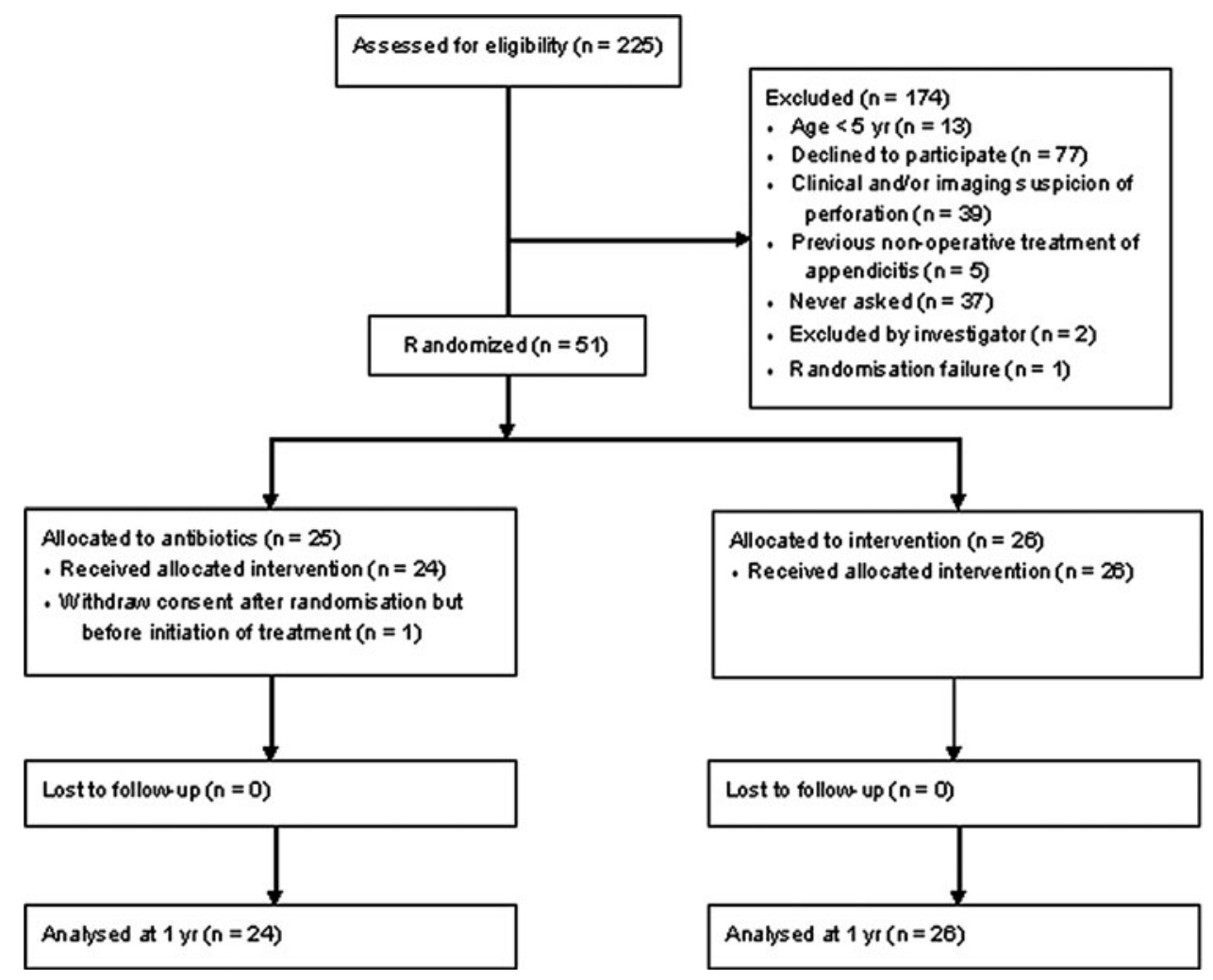

FIGURE 1. Study flowchart.

TABLE 1. Comparison of Participants, Those Eligible but Not Enrolled and Those Not Invited to Participate

\begin{tabular}{|c|c|c|c|c|c|}
\hline & $\begin{array}{c}\text { Randomized } \\
\text { Children }(\mathrm{n}=\mathbf{5 0}) \\
\end{array}$ & $\begin{array}{c}\text { Declined to } \\
\text { Participate }(n=77)\end{array}$ & $P^{*}$ & $\begin{array}{c}\text { Not Invited to } \\
\text { Participate }(n=37) \\
\end{array}$ & $P \dagger$ \\
\hline Age, yr & $11.2(5.9-15.0)$ & $11.0(5.8-14.9)$ & 0.369 & $10.8(5.3-14.9)$ & 0.268 \\
\hline Male sex, n (\%) & $26(52)$ & $42(55)$ & 0.779 & $23(62)$ & 0.345 \\
\hline $\begin{array}{l}\text { Duration of symptoms }<48 \mathrm{~h} \text {, } \\
\text { n (\%) }\end{array}$ & $43(86)$ & $61(79)$ & 0.332 & $25(68)$ & 0.04 \\
\hline $\mathrm{CRP}$ at admission, $\mathrm{mg} / \mathrm{L}$ & $28(1-185)$ & $19(1-152)$ & 0.414 & $17.5(1.0-150.0)$ & 0.909 \\
\hline WBC $\left(\times 10^{9} / \mathrm{L}\right)$ at admission & $14.3(4.5-26.9)$ & $15.0(5.2-27.2)$ & 0.086 & $15.0(6.1-33.5)$ & 0.297 \\
\hline $\begin{array}{l}\text { Neutrophils }\left(\times 10^{9} / \mathrm{L}\right) \text { at } \\
\text { admission }\end{array}$ & $11.5(2.5-23.5)$ & $12.5(1.5-24.0)$ & 0.155 & $3.6(12.5-30.1)$ & 0.295 \\
\hline Temperature at admission, ${ }^{\circ} \mathrm{C}$ & $37.4(36.3-39.0)$ & $37.3(35.9-37.3)$ & 0.177 & $37.1(35.7-39.3)$ & 0.392 \\
\hline
\end{tabular}

Data are median (range) unless specified.

${ }^{*}$ Comparison between randomized children and those who declined to participate.

$\dagger$ Comparison between randomized children and those who were not invited to participate.

CRP indicates C-reactive protein; WBC, white blood cells.

need to perform an appendectomy in children with longer symptom duration. The surgery and nonoperative treatment groups had similar demographic and admission characteristics (Table 2). All patients had at least 1 ultrasound examination, 1 had a second ultrasound scan, and 4 had a CT scan after the initial ultrasound scan. The reason for repeated examination was, in all cases, that the appendix was not seen at the initial examination.

\section{Primary Outcome}

All children randomized to surgery had a laparoscopic appendectomy with a 3-port technique. Histological examination confirmed the diagnosis of acute appendicitis in all cases (ie, no negative appendectomy, 21 phlegmonous appendicitis, 3 gangrenous appendicitis, and 2 perforated appendicitis), and there were no significant complications in this group.

All children randomized to nonoperative treatment with antibiotics received antibiotics per protocol. Two of these children had a significant complication. One child underwent an early appendectomy on day 2, as symptoms had failed to improve; a macroscopically normal appendix was removed and the child had a diagnosis of mesenteric lymphadenitis. Histological examination of the appendix was normal. This patient had had an inconclusive ultrasound scan and a CT scan suggestive of appendicitis with a tubular structure measuring 9 to $10 \mathrm{~mm}$. The final report on this CT scan (produced after the surgery) was changed to a negative investigation. A second child returned to the emergency department on day 9 after randomization 
TABLE 2. Comparison of Treatment Groups

\begin{tabular}{|c|c|c|c|}
\hline & \multicolumn{3}{|c|}{ Randomized Children } \\
\hline & Surgery $(n=26)$ & $\begin{array}{c}\text { Nonoperative } \\
\text { Treatment }(n=24)\end{array}$ & $\boldsymbol{P}$ \\
\hline Age, yr & $11.1(6.2-14.8)$ & $12.2(5.9-15.0)$ & 0.130 \\
\hline Male sex, n (\%) & $12(46)$ & $14(58)$ & 0.389 \\
\hline Duration of symptoms $<48 \mathrm{~h}, \mathrm{n}(\%)$ & $23(88)$ & $20(83)$ & 0.602 \\
\hline $\mathrm{CRP}$ at admission, $\mathrm{mg} / \mathrm{L}$ & $27.0(1.0-175.0)$ & $30.5(1.0-185.0)$ & 0.892 \\
\hline WBC $\left(\times 10^{9} / \mathrm{L}\right)$ at admission & $14.5(4.5-26.9)$ & $14.0(4.8-19.0)$ & 0.918 \\
\hline Neutrophils $\left(\times 10^{9} / \mathrm{L}\right)$ at admission & $11.6(2.9-23.5)$ & $11.5(2.5-16.8)$ & 1.0 \\
\hline Temperature at admission, ${ }^{\circ} \mathrm{C}$ & $37.5(36.5-38.5)$ & $37.3(36.6-39.0)$ & 0.199 \\
\hline
\end{tabular}

Data are median (range) unless specified.

CRP indicates C-reactive protein; WBC, white blood cells.

with moderate abdominal pain after initial successful antibiotic treatment according to the study protocol. Ultrasound scan revealed signs of ongoing inflammation, and a walled-off perforated appendicitis was found at laparoscopic appendectomy. The primary outcome was similar in each group [appendectomy group 26/26 (100\%) vs nonoperative treatment group $22 / 24(92 \%) ; P=0.23$ ].

\section{Secondary Outcomes}

During the 1-year follow-up period, there were no significant or minor complications in the surgery group. In the nonoperative treatment group, there were no minor complications. However, 1 child had appendectomy for histopathologically confirmed recurrent acute appendicitis 9 months after randomization and 1 asymptomatic child underwent (histopathologically normal) appendectomy at parental request. A further 5 children returned with mild abdominal pain and had laparoscopic appendectomies at surgeon and parental discretion. All had a varying degree of fibrosis in the appendix but no inflammation. In all cases, symptoms resolved after surgery. Therefore, after 1 -year of follow up, 15 of 24 children (62\%) randomized to primary antibiotic treatment had not undergone an appendectomy.

Twelve children had a diagnosis of an appendicolith on imaging, 7 of 26 in the surgery group and 5 of 24 in the nonoperative treatment group $(P=0.74)$. Of the 5 children with an appendicolith in the nonoperative treatment group, 3 had appendectomy (none as primary failures, 1 due to recurrent acute appendicitis, 1 due to recurrent symptoms without appendicitis, and 1 on parental request). Thus, in the nonoperative treatment group, 2 children with an appendicolith did not have appendectomy within 1 year of follow-up, and of the total 9 who have had an appendectomy, only 3 had an appendicolith on imaging at the initial presentation.

Time from randomization to actual discharge home was calculated for each participant. The median time to discharge was significantly shorter in the surgical group [34.5 (16.2-95.0) hours] than in the nonoperative treatment group [51.5 (29.9-86.1) hours] $(P=$ $0.0004)$. Despite this, the cost for the initial inpatient stay was significantly lower for the nonoperative treatment group [30,732 $(18,980$ $63,863) \mathrm{SEK}]$ than for the surgery group $[45,805(33,042-94,638)$ SEK] $(P<0.0001)$.

The total cost of treatment, including the cost of those patients having an appendectomy during the follow-up period, was similar in both treatment groups [nonoperative treatment 34,587 $(19,120$ $146,552)$ SEK vs surgery $45,805(33,042-94,638)$ SEK $](P=0.11)$.

\section{DISCUSSION}

In this pilot RCT comparing nonoperative treatment with antibiotics and surgery for acute nonperforated appendicitis in children, we have shown that nonoperative treatment is feasible and safe. Over- all, $40 \%$ of families asked to participate accepted and were enrolled, suggesting that nonoperative treatment is of interest to this patient population and their families. We consider it possible that in future randomized trials in children, this consent rate might be improved, as during the study we were unable to provide the parents with any evidence of safety or efficacy of antibiotics alone whereas future studies would have such evidence from this pilot trial. On the basis of the recruitment rate achieved, we believe a future RCT would be feasible.

Although this pilot trial was not adequately powered to detect differences in treatment efficacy, outcome data are useful to inform future studies. As defined, effective treatment was achieved in $100 \%$ and $92 \%$ in the surgery and nonoperative treatment groups, respectively. In the nonoperative treatment group, only 2 of 24 patients failed to meet criteria for the primary endpoint. One of them had mesenteric lymphadenitis, which may explain the failure to respond to antibiotics, as this patient's condition did not improve by antibiotic treatment. The other returned after initial resolution in symptoms with antibiotics and was found to have perforated appendicitis.

An important consideration for surgeons and parents after successful nonoperative treatment of acute appendicitis is the fate of the appendix. In this study, we did not offer routine interval appendectomy. A potential benefit of nonoperative treatment is the avoidance of an appendectomy (and associated general anesthesia) at all. For this benefit to be realized, the recurrent appendicitis rate must be low and acceptable to both surgeons and parents. In this study, there was one case of histologically proven recurrent appendicitis during the follow-up period (5\%). However, a further 6 children had appendectomy within the 1-year follow-up period for reasons other than recurrent acute appendicitis including one at parental request. As this was a pilot trial of a novel treatment strategy (antibiotics for acute appendicitis in children), we were liberal with regard to indications for surgery during the follow-up period among children in the nonoperative treatment group. It is possible that patients in this group would not have had surgery if they had presented with their symptoms outside the trial setting. This may have contributed to the high rate of surgery during follow-up and raises the important question of what is an appropriate threshold for appendectomy in children who have been successfully discharged home after nonoperative treatment.

For nonoperative treatment to be considered equivalent to appendectomy, some may believe that the length of hospitalization should be similar. In this pilot trial, the postrandomization length of stay was longer for children in the nonoperative treatment group than for children undergoing appendectomy. A possible explanation for this is that we stipulated a minimum of 48 hours of intravenous antibiotics in our protocol. In the future, it may be possible to reduce this duration without affecting efficacy. During analysis of these time-related data, it became apparent that significant delays 
between randomization and surgery will impact on the time from randomization to discharge and therefore potentially influence the interpretation of this outcome measure. Delays between randomization and surgery may occur due to hospital workload and/or time of presentation, as typically appendectomy is no longer performed during the night. Median time between randomization and surgery in this study was 5.8 hours but with a range of 0.8 to 26.2 hours. These factors must be considered carefully in any future RCT.

Although overall cost was similar between the 2 treatment groups, the cost of the initial inpatient treatment was significantly higher in the surgery group. Thus, the additional admissions for recurrent symptoms in the nonoperative treatment groups were a significant determinant of cost in this group. A cost-effectiveness analysis should be performed as part of any future study.

Although the number of patients treated nonoperatively was small, there were no safety issues either during the acute admission or during the follow-up period and so this trial provides no evidence that nonoperative treatment of acute appendicitis is unsafe. As this was a pilot trial with a relatively small sample size, the efficacy data produced should be interpreted with caution. Importantly, we do not recommend nonoperative treatment of simple acute appendicitis in all children until further large-scale efficacy studies have been completed. This pilot trial suggests that nonperforated acute appendicitis in children may be safely treated with antibiotics and that it would be appropriate and feasible to proceed to a similar larger, RCT to determine the efficacy of nonoperative treatment in this population.

\section{REFERENCES}

1. Svensson JF, Hall NJ, Eaton S, et al. A review of conservative treatment of acute appendicitis. Eur J Pediatr Surg. 2012;22:185-194.

2. Wilms IM, de Hoog DE, de Visser DC, et al. Appendectomy versus antibiotic treatment for acute appendicitis. Cochrane Database Syst Rev. 2011;9:CD008359.

3. Blakely ML, Williams R, Dassinger MS, et al. Early vs interval appendectomy for children with perforated appendicitis. Arch Surg. 2011;146:660-665.

4. Holcomb GW, III, St Peter SD. Current management of complicated appendicitis in children. Eur J Pediatr Surg. 2012;22:207-212.

5. Abes M, Petik B, Kazil S. Nonoperative treatment of acute appendicitis in children. J Pediatr Surg. 2007;42:1439-1442.

6. Schulz KF, Altman DG, Moher D. CONSORT 2010 statement: updated guidelines for reporting parallel group randomised trials. BMJ. 2010;340: c332.

7. Moher D, Hopewell S, Schulz KF, et al. CONSORT 2010 explanation and elaboration: updated guidelines for reporting parallel group randomised trials. BMJ. 2010;340:c869. 